\title{
Extra nodal natural killer lymphoma mimicking canine fossa infection - a clinical report
}

\begin{abstract}
Maxillo facial region is the second most common site for presentation of extra nodal lymphomas, among these extra nodal variant of natural killer $t$ cell lymphoma (ENKTCL) is newly recognized entity in WHO classification1.In this report, we describe the clinical and pathologic features of an unusual aggressive lymphoid neoplasm in a 48 year male patient who presented with progressively increasing swelling of the right side of the face, fever, halitosis, uncontrolled diabetes mellitus and chronic alcoholism. We would like to share the diagnostic dilemmas and role of immuno phenotyping in the diagnosis of such conditions. Although our efforts to treat the patient was futile, a high index of suspicion for this tumor is needed when an angiocentric lesion is found and a thorough immuno histological study should always be conducted in such cases.
\end{abstract}

Keywords: extra nodal lymphomas; natural killer t- cell lymphoma; canine space infections
Volume 9 Issue I - 2018

\section{Praveen Kumar S, Vikas Dhupar, Francis \\ Akkara}

Department of Oral Maxillo Facial Surgery, Goa Dental College

and Hospital, India

Correspondence: Praveen Kumar S, Fellow AOCMF (Austria), Certified by International Board of OMFS, Assistant Professor, Department of Oral Maxillo Facial Surgery, Goa Dental College and Hospital, Bambolim, Goa, 403202, India,Tel 91-9881954606, Email praveen1931@gmail.com

Received: February 06, 2017 | Published: February 01, 2018

\section{Introduction}

Mature T- or natural killer (NK)/T-cell lymphoma makes up only $5 \%$ to $18 \%$ of all cases of non-Hodgkin lymphoma (NHL). The relative frequency and optimal therapy for these disorders is not well defined. Extra nodal NK/T-cell lymphoma is a distinct entity in the World Health Organization (WHO) classification. ${ }^{1}$ It is more prevalent in Asians and Native Americans, occurs most often in adults, and almost always occurs in extranodal sites with a predilection for the upper airway including nasal passages, paranasal sinuses, and nasopharynx. ${ }^{2,3}$ Extranasal sites include skin, soft tissue, and the gastrointestinal tract. Extranodal (NK)/T-cell lymphoma nasal type is a rare entity with an extremely aggressive nature. We report a unique case of extranodal NK/T-cell lymphoma, nasal type, in a 48-yearold Indian male presenting with progressively increasing hemi facial swelling, periorbital edema and uncontrolled diabetes mellitus mimicking Orofacial infection. Our case demonstrates how the acute presentation of an aggressive extranodal NK/T-cell lymphoma, nasal type, can present in a similar fashion to canine space swelling. Through this report we would like to stress on considering an occult malignancy as differential diagnosis in individuals with altered immune function not responding to conventional treatment and also the role of immuno histochemistry in diagnosing such aggressive tumors.

\section{Case presentation}

A 48 year old male patient was referred to our outpatient department with history of unsettling facial swelling and uncontrolled diabetes mellitus for which he was hospitalized under the general medicine ward. History revealed that the patient had a fall from the bed in an inebriated state three days prior, following which he developed swelling over the right side of the face and pain in the upper front teeth. In the mean time he was treated by his dentist who extracted the upper right canine and premolar presuming that the swelling in the canine fossa was caused by dental infection. An intra oral incision and drainage was also performed in order to drain the abscess. On clinical examination he presented with right -sided infra orbital swelling with a portion of the overlying skin necrosed. The swelling was seen obliterating the naso labial fold on the same side. Gross edema of the peri-oral and periorbital tissue with resultant narrowing of the palpebral fissure was noted on the same side (Figure1). Intraoral examination revealed an extraction socket in relation to upper right canine and premolar and vestibular laceration with slough and poor oral hygiene (Figure 2).

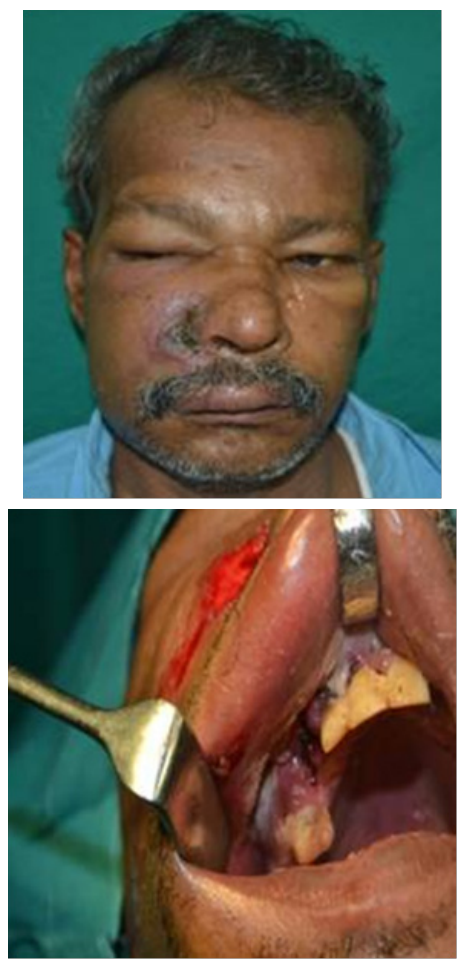

The patient was started on intravenous antibiotics which included Piperacillin $2.5 \mathrm{Gms}$ and Linezolid 600mg 12 hourly. A combination of Injection Human Actrapid and Insultard were used for his glyceamic control. However, he did not respond to the therapy and the 
area was severely infected. CT scan revealed the presence of a diffuse soft tissue swelling superficial to the right parotid space extending to the right buccal space with increased density and streakiness of sub cutaneous fat consistent with inflammation. The underlying bone seemed to be unaffected (Figure 3).

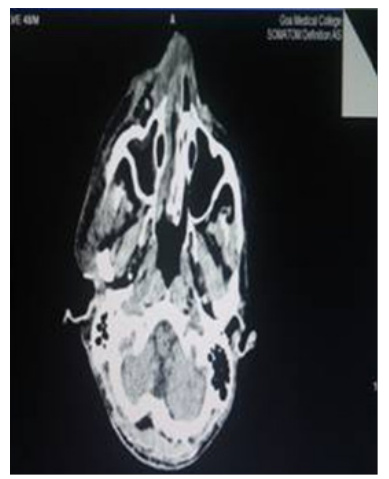

Intraoral site was explored under local anesthesia for debridement of the slough and also to obtain tissue for histopathological analysis. Surgical exploration of the site revealed the presence of peanut sized gauze which was deeply lodged in the wound. The H\&E stained revealed extensive necrosis with proliferating plump myofibroblasts admixed with a moderate inflammatory cell infiltrate which were chiefly lymphocytic and suggestive of inflammatory myofibroblastic pseudo tumor. The report also suggested the presence of fungal colonies which were PAS positive. Finally the patient underwent wide local excision of the lesion and overlying necrosed skin under general anesthesia presuming it to be a pseudo tumor with super imposed fungal infection. The specimen was sent for histopathological and immunohisto chemical analysis. The H\&E stained sections showed extensive necrosis, areas of hemorrhage, prominent blood vessels and multifocal cellular infiltration (Figure 4). Some of the vessels were thrombosed, and showed perivascular coagulative necrosis. The major cellular component was atypical lymphoid cells with evidence of super imposed fungal infection. Immuno histochemistry report were suggestive of a Non-Hodgkin's lymphoma of NK/T cell, nasal type with tumor cells expression of CD3( weak), CD 2 (weak), CD 56 and granzyme B. In situ hybridization of EBV RNA was positive. (SRL Diagnostics, Mumbai). There was considerable delay in procuring the immunohistochemical reports, so the patient was started on systemic antifungal therapy with injection Amphotericin B. The patient's general condition progressively worsened with recurrent bouts of intra oral and nasal hemorrhage. His glycemic control proved to be a challenge despite best efforts by the endocrinologist. The patient finally died of the disease within a week of admission before any other form of treatment could be instituted.

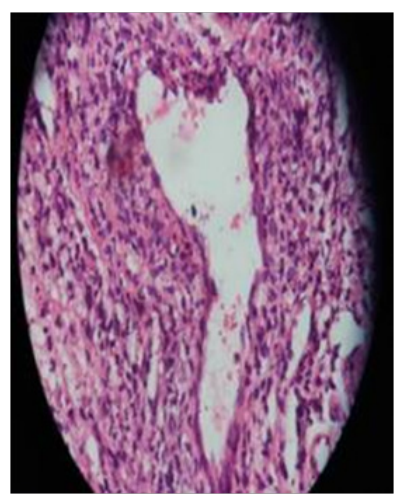

\section{Discussion}

Malignant lymphomas of the head and neck region which originate from oral and paranasal tissues form an important clinical entity which often present as clinical and histological challenge for early diagnosis and management. ${ }^{4}$ Among these lymphomas the extra nodal NK/T-cell lymphoma, nasal type, represents a group of distinct neoplasm that are more common in Asia and Latin America. ${ }^{3,4}$ The diagnosis of these cases have posed significant problem to both clinician and pathologists in the past. In the above discussed case the patient presented with a history of trauma with acute onset of swelling and uncontrolled diabetes mellitus which mislead us to arrive at a proper early diagnosis. The presentation of extensive necrosis in this tumor frequently misleads the pathologists to consider it an infectious process, especially when bacterial colonies or fungi are identified. It is evident that diagnosis of these lymphomas is dependent on a series of laboratory tests, special staining techniques and immuno histochemical analysis for the relevant markers. A definitive diagnosis of nasal NK/T-cell lymphoma depends on immunophenotyping. The major immunophenotype for extranodal NK/T-cell lymphoma, nasal type, is $\mathrm{CD} 56+$, surface CD37 and cytoplasmic CD3e+. ${ }^{5-7}$ The treatment of extranodal NK/T-cell lymphoma, nasal type, is difficult and complex. Some authors consider that surgery is ineffective and may even cause deterioration of the lesions by inducing rapid progression of the disease. Surgical resection of the lesions has been proposed, essentially for diagnostic purposes, but also to promote drainage of necrotic cavities. During the last few years several treatment options have been proposed for patients with ENKTCL and current evidence relies on retrospective studies and series reports, ranging from radiotherapy alone to high-dose chemotherapy. ${ }^{8}$

\section{Conclusion}

Since these tumors are usually angioinvasive and angiodestructive, it frequently induces extensive tissue necrosis with superimposed bacterial or fungal infections that may mask the true neoplasm. Due to the aggressive nature of this tumor, a misdiagnosis of an infectious process may lead to a disastrous outcome for the patient. Thus the recognition of this entity is of great clinical significance, in instituting the required treatment at the earliest.

\section{Funding}

None.

\section{Acknowledgements}

None.

\section{Conflicts of interest}

None.

\section{References}

1. Dalal Abdullah Al-Hakeem, Stefano Fedele, Roman Carlos, et al Extranodal NK/T-Cell Lymphoma, Nasal Type. Oral Oncology. 2007;43(1):4-14.

2. Ko YH, Cho EY, Kim JE, et al. NKand NK-like T-cell lymphoma in extranasal sites: A comparative clinicopathological study according to site and EBV status. Histopathology. 2004;44(5):480-489.

3. Chan JKC, Sin VC, Wong KF, et al. Nonnasal lymphoma expressing the natural killer cell marker CD56: A Clinicopathologic Study of 49 cases of an uncommon aggressive neoplasm. Blood. 1997;89(12):4501-4513. 
4. Kinney MC. The role of morphologic features, phenotype,genotype, and anatomic site in defining extranodal T-cell or NK-cell neoplasms. Am J Clin Pathol. 1999;111(Suppl 1):S104-S118.

5. Califano L, Zupi A, Maremonti P, et al. Sinonasal lymphoma presenting as a lethal midline granuloma.Case Report. J Oral Maxillofac Surg. 1998;56(5):667-671.

6. Suzumiya J, Takeshita M, Kimura N, et al. Expression of adult and fetal natural killer cell markers in sinonasal lymphomas. Blood. 1994;83(8):2255-2260.
7. Ko YH, Ree HJ, Kim WS, et al. Clinicopathologic and genotypic study of extranodal nasal-type natural killer/T-cell lymphoma and natural killer precursor lymphoma among Koreans. Cancer. 2000;89(10):2106-2116.

8. Kim WS, Song SY, Ahn YC, et al. CHOP followed by involved field radiation: is it optimal for localized nasal natural killer/T-cell lymphoma? Ann Oncol. 2001;12(3):349-352. 